\title{
Can Therapeutic-Range Chronic Phenytoin Administration Cause Cerebellar Ataxia?
}

Review Article

Journal of Epilepsy Research pISSN 2233-6249 / eISSN 2233-6257

Received January 17, 2017

Accepted March 13, 2017

Corresponding author: Beomseok Jeon Department of Neurology, College of Medicine, Seoul National University, 101 Daehak-ro, Jongno-gu, Seoul 03080, Korea

Tel. $+82-2-2072-2114$

Fax. +82-2-744-1785

E-mail; brain@snu.ac.kr

\author{
Hye-Jin Moon ${ }^{1}$, Beomseok Jeon ${ }^{2}$ \\ ${ }^{1}$ Department of Neurology, Keimyung University Dongsan Medical Center, Daegu; ${ }^{2}$ Department of Neurology and \\ Movement Disorder Center, Seoul National University Hospital, Seoul, Korea
}

\begin{abstract}
Phenytoin (PHT) is a first line antiepileptic drug (AED) used to treat many epilepsy syndromes. As with other AEDs, there are various adverse effects associated with PHT. For this brief review, we searched for evidence of cerebellar ataxia as a chronic adverse effect of therapeutic-range PHT treatment. Many previous studies appeared related to this issue, but many of those studies were designed to resolve questions related to the persistent residual effects of toxic-range PHT therapy, or they were inconclusive due to an absence of critical information such as PHT serum concentration, cerebellar symptoms/signs, and other factors contributing to cerebellar degeneration. Nevertheless, there were a few reports suggesting that cerebellar ataxia may be a chronic adverse effect of therapeutic-range PHT therapy and that a possible pathomechanism for that effect is folate deficiency. Moreover, there is the possibility that there may be patient-specific susceptibility factors affecting ataxia. Further studies are needed to elucidate the incidence, risk factors, and pathomechanism of cerebellar ataxia as a chronic adverse effect of therapeutic-range PHT treatment. (2017;7:21-24)
\end{abstract}

Key words: Phenytoin, Antiepileptic drug, Adverse effect, Cerebellar ataxia

\section{Introduction}

Hydantoins are derivatives of a common five-membered heterocyclic ring that differ according to various combinations of alkyl and phenyl substitutions at three positions on the ring. In the late 1930s, Putnam and Merritt tested several hydantoin derivatives and found phenytoin (diphenylhydantoin, PHT) to have a promising antiepileptic effect with acceptable sedative properties. ${ }^{1}$ In human trials, PHT was shown to be effective ${ }^{2}$ and thereafter became accepted as a first line antiepileptic drug (AED) for many epilepsy syndromes.

As with other AEDs, adverse effects of PHT have been identified and some may lead to drug discontinuation. Well-known dose-related reversible adverse effects of PHT include nystagmus, ataxia, diplopia, and drowsiness. Ataxia related to PHT therapy usually involves station and gait rather than fine motor movements. There is a relationship between PHT intoxication symptoms and drug concentration and, in the majority of patients, ataxia appears at a PHT plasma concentration of approximately $30 \mathrm{ug} / \mathrm{mL}$. $^{3}$ However, there are a few reports of cerebellar ataxia being a chronic adverse effect of therapeutic-range PHT treatment.

\section{Experimental studies}

Most previous experimental PHT studies have investigated chronic cerebellar dysfunction following acute PHT toxicity. ${ }^{4-10}$ These studies were designed to evaluate the effect of toxic-level PHT administration during a relatively short period (longest duration was six months). Several of the studies revealed Purkinje cell loss as well as other evidence of cerebellar degeneration in various animals given toxic levels of PTH..$^{4-8}$ Dam $^{9}$ criticized these experimental studies, including his earlier study in pigs, ${ }^{10}$ suggesting that the reported abnormalities may have been the result of fixation artifacts, hypoxic lesions, or failure to use quantitative Purkinje cell counting methods rather than the result of true cerebellar degeneration due to PTH. Thus far, experimental studies have not elucidated PHT's role in cerebellar dysfunction or degeneration in subjects undergoing chronic administration of therapeutic-range PTH.

No experimental study reports on cerebellar dysfunction related to chronic use of PHT within the therapeutic range. Therefore, that relationship remains undetermined, and it is clear that further studies are required not only to resolve the differing views about chronic cerebellar degeneration after acute toxicity, but also to determine the types and incidences of cerebellar dysfunction after long-term

This is an Open Access article distributed under the terms of the Creative Commons Attribution Non-Commercial License (http://creativecommons.org/licenses/by-nc/3.0/) which permits unrestricted non-commercial use, distribution, and reproduction in any medium, provided the original work is properly cited. 
therapeutic-range PHT treatment.

\section{Clinical studies}

Previous case reports and case series have described cerebellar degeneration in patients taking $\mathrm{PHT} .{ }^{11-19}$ However, most of those reports had one or more weaknesses that did not permit determination of the relationship between cerebellar ataxia and chronic PHT therapy within the therapeutic range. For example, patients in some studies had other possible causes of cerebellar degeneration and ataxia such as generalized tonic-clonic seizures, hypoxia from other causes (encephalitis or electroconvulsive therapy), chronic concomitant use

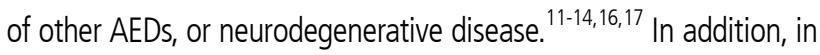
most cases the daily PHT dosage or PHT serum level was above the therapeutic range, ${ }^{11,17,19}$ and in many reports the authors assessed the irreversible or persistent toxic effects of PHT after acute intoxication. Thus, these studies could not address our primary question-Can chronic phenytoin administration within therapeutic range cause cerebellar ataxia? Neither ataxia and cerebellar degeneration nor ataxia and cerebellar atrophy are synonymous. Cerebellar degeneration and cerebellar atrophy have been observed in patients with chronic therapeutic-range PHT therapy. In some reports, ${ }^{11,13}$ either the patients did not show cerebellar ataxia or the authors did not mention cerebellar symptoms or signs. Finally, PHT therapy within the therapeutic range cannot be equated to PHT therapy with commonly used dosages. Due to the pharmacokinetic characteristics of $\mathrm{PHT}$, including first-order kinetics, ${ }^{20}$ common drug-drug interactions due to competitive protein-bound properties, ${ }^{21,22}$ and the metabolic dependency of the cytochrome p450 system, ${ }_{1}^{23}$ even when applying commonly used dosages the serum level of PHT can fluctuate and may increase to the toxic range. However, in many previous reports, ${ }^{11,13,14,16}$ PHT serum level data are absent. Thus, to address our primary question, there is a need to evaluate serum PHT concentrations or, if needed, unbound PHT concentrations.

Several autopsy cases ${ }^{8,11-13,15}$ have demonstrated cerebellar degeneration only rather than concomitant cell loss in the cerebellum, hippocampus, and other structures sensitive to hypoxia. The authors of those studies implicate PHT rather than hypoxia as the cause of the degeneration. However, subjects in some of those studies had been treated with high PHT dosages ${ }^{11}$ or had undergone short duration PHT treatment. ${ }^{13}$ For some subjects in those studies there was no information on PHT serum concentrations. ${ }^{13}$ Some rare cases exhibited persistent cerebellar damage attributable to long-term PHT treatment. ${ }^{8,11,12,15}$ Among those studies, two reports mentioned cerebellar manifestations. ${ }^{12,15}$ In the biopsy study reported by Salcman et al. ${ }^{24}$ cerebellar signs were rare among patients with marked Purkinje cell layer degeneration. Such studies indicate that the cerebellum may be able to compensate for cell loss induced by PHT treatment. Moreover, there is a possibility that cerebellar degeneration observed by autopsy or biopsy does not indicate the presence of cerebellar symptoms such as ataxia.

Neuroimaging studies of PHT treated patients, using computed tomography (CT) or magnetic resonance imaging (MRI), have been conducted. McLain et al. ${ }^{19}$ reported on five patients undergoing chronic PHT therapy with cerebellar dysfunction and cerebellar atrophy on CT scans. All had high plasma PHT levels and none had seizures when the cerebellar symptoms appeared. The authors suggested that a chronic exposure of high level PHT therapy rather than seizures was responsible for the cerebellar degeneration. Luef et al. ${ }^{25}$ reported similar results from their 1.5 T MRI study. They observed 11 patients undergoing chronic PHT therapy (> 1 year duration) and experiencing episodes of abnormally increased serum PHT levels. Most of their patients developed clinical signs of cerebellar dysfunction: five patients had normal cerebellar structures whereas six had moderate to severe cerebellar atrophy. However, because of the high serum PHT levels in all of the patients in those studies, the results do not apply to our primary question.

Koller et al. ${ }^{26}$ studied 55 cases of cerebellar atrophy that were identified by using CT. Among those cases, eight patients had been taking PHT at a therapeutic level for an average of 12 years. Those patients showed significant superior cerebellar cistern, cerebellopontine angle cistern, and cisterna magna enlargement. However, there was no evidence of cerebellar dysfunction. Their results are similar to previous autopsy/biopsy studies that showed divergent clinical manifestation and pathologic results.

Ney et al. ${ }^{27}$ and De Marcos et al. ${ }^{28}$ evaluated cerebellar atrophy in epileptic patients with long-term PHT exposure by using MRI (1.0 T and $2.0 \mathrm{~T}$, respectively). Ney and coworkers ${ }^{27}$ reported that their patient group had a significantly high frequency of cerebellar atrophy. However, they did not describe any cerebellar symptoms or signs, and they did not evaluate serum PHT concentrations. De Marcos ${ }^{28}$ performed cerebellar volumetry and detected cerebellar atrophy (i.e., cerebellar volumes less than two standard deviations below the mean of the control group) in $35.7 \%$ of the patients. Their indicator of cerebellar atrophy correlated with epilepsy duration and years of $\mathrm{PHT}$ treatment, but not with the frequency of generalized seizures, 
age, or mean daily PHT dosage. They did not evaluate patients' cerebellar symptoms or signs. Moreover, serum PHT levels were available only in approximately one third of their patients, and half of those had toxic serum PHT levels.

The report by Munoz-Garcia et al. ${ }^{29}$ focused on ataxia in chronic antiepileptic treatment. They studied 95 epileptic patients on chronic antiepileptic treatment including those undergoing PHT therapy $(81 \%)$. Twenty-eight $(29.5 \%)$ of the patients with truncal ataxia had longer treatment periods, received a significantly larger variety of drugs, and had higher serum phenobarbital levels than the symptom-free group. Those authors suggested that cerebellar ataxia is attributed to chronic multiple-drug antiepileptic therapy rather than PHT-only treatment.

In conclusion, the occurrence of cerebellar ataxia in patients undergoing chronic PHT therapy within the therapeutic range is possible, but studies with results relevant to our primary question are rare and inconclusive. Therefore, further well-designed, large sample size studies are needed.

\section{Possible mechanisms}

The pathomechanism of toxicity due to chronic $\mathrm{PHT}$ administration is unclear. Some researchers have suggested folate deficiency as a possible mechanism because PHT inhibits folate absorption from folate polyglutamates, a major component of food folate, and inhibits the activity of folate conjugase from human jejunal mucosa at pharmacological concentrations in vitroand in vivo. ${ }^{30}$ Muños et al. ${ }^{29}$ suggested that folate depletion is associated with the induction of ataxia by chronic anticonvulsant treatment. In their report, serum folate levels were significantly lower in the ataxic group than in the control group. They proposed two possible mechanisms. First, decreased folate levels may impair hepatic drug-metabolizing capacity ${ }^{31}$ and thus increase serum drug levels after a long, steady-state, symptom-free period. Alternatively, folate deficiency might directly impair neural functioning.

\section{Conclusion}

For this brief review, we searched for evidence indicating that cerebellar ataxia was a chronic adverse effect of therapeutic-range PHT therapy. There are several previous studies related to this issue, but upon detailed examination we found that many of those studies were designed to resolve other questions related to the persistent re- sidual effect of toxic-range PHT therapy or were inconclusive due to an absence of critical information on other factors such as PHT serum concentration, cerebellar symptoms/signs, and other factors contributing to cerebellar degeneration. Nevertheless, there were a few reports suggesting that cerebellar ataxia may be a chronic adverse effect of therapeutic-range PHT therapy and that a possible pathomechanism for that effect was folate deficiency. Moreover, there may be patient susceptibility factors affecting ataxia. Further studies are needed to elucidate the incidence, risk factors, and pathomechanism of cerebellar ataxia as a chronic adverse effect of therapeutic-range PHT treatment.

\section{Key points}

- Ataxia is one of the well-known dose-related reversible adverse effects of PTH therapy.

- Most experimental studies have investigated chronic cerebellar dysfunction following acute intoxication of PHT.

- Most case reports/series were inconclusive due to an absence of some critical information.

- Several autopsy cases and neuroimage studies have demonstrated cerebellar degeneration but cerebellar signs were rare among patients with marked degeneration/atrophy.

- Further studies about the incidence, risk factors, and pathomechanism of cerebellar ataxia as a chronic adverse effect of therapeutic-range PHT treatment are needed.

\section{Conflict of Interest}

None of the authors has any conflict of interest to disclose. We confirm that we have read the Journal's position on issues involved in ethical publication and affirm that this report is consistent with those guidelines.

\section{References}

1. Friedlander WJ. Putnam, Merritt, and the Discovery of Dilantin $\AA$. Epilepsia 1986;27 Suppl 3:S1-20.

2. Merritt HH, Putnam TJ. Sodium diphenyl hydantoinate in the treatment of convulsive disorders. JAMA 1938;111:1068-73.

3. Kutt H. Phenytoin: relation of plasma concentration to seizure control. In Antiepileptic drugs. 2nd Ed. New York: Raven, 1982:241-6.

4. Perez del Cerro M, Snider RS. Studies on Dilantin intoxication I. Ultrastructural analogies with the lipoidoses. Neurology 1967;17:452-66. 
5. Kokenge $R$, Kutt $H$, McDowell F. Neurological sequelae following dilantin overdose in experimental animals. Neurology 1965;15:823-9.

6. Snider RS, Perez del Cerro M. Drug-induced dendritic sprouts on Purkinje cells in the adult cerebellum. Exp Neurol 1967;17:466-80.

7. Snider R, Del Cerro M. Diphenylhydantoin-proliferating membranes in cerebellum resulting from intoxication. In Antiepileptic drugs. New York: Raven, 1972:237-45.

8. Utterback RA. Parenchymatous cerebellar degeneration complicating diphenylhydantoin (Dilantin) therapy. Arch Neurol Psychiat 1958;80:180-1.

9. Dam M. The density and ultrastructure of the Purkinje cells following diphenylhydantoin treatment in animals and man. Acta Neurol Scand Supp/ 1972;49:3-65.

10. Dam M. Organic changes in phenytoin-intoxicated pigs. Acta Neurol Scand 1966;42:491-4.

11. Hofmann WW. Cerebellar lesions after parenteral dilantin administration. Neurology 1958:8:210-4.

12. Ghatak NR, Santoso RA, McKinney WM. Cerebellar degeneration following long-term phenytoin therapy. Neurology 1976;26:818-20.

13. Rapport II RL 2nd, Shaw CM. Phenytoin-related cerebellar degeneration without seizures. Ann Neurol 1977;2:437-9.

14. Selhorst JB, Kaufman B, Horwitz SJ. Diphenylhydantoin-induced cerebellar degeneration. Arch Neurol 1972;27:453-5.

15. Haberland C. Cerebellar degeneration with clinical manifestation in chronic epileptic patients. Psychiatr Neurol (Basel) 1962;143:29-44.

16. Afifi AK, Van Allen MW. Cerebellar atrophy in epilepsy. Pneumographic and histological documentation of a case with psychosis. J Neurol Neurosurg Psychiatry 1968;31:169-74.

17. Logan WJ, Freeman JM. Pseudodegenerative disease due to diphenylhydantoin intoxication. Arch Neurol 1969;21:631-7.

18. Lusins JO, Jutkowitz R. Residual cerebellar systems dysfunction and peripheral neuropathy after diphenylhydantoin therapy. Mt Sinai J Med 1972;39:617-21.

19. McLain LW Jr, Martin JT, Allen JH. Cerebellar degeneration due to chronic phenytoin therapy. Ann Neurol 1980;7:18-23.

20. Richens A, Dunlop A. Serum-phenytoin levels in management of epilepsy. Lancet 1975;2:247-8.

21. Haidukewych D, Rodin EA, Zielinski JJ. Derivation and evaluation of an equation for prediction of free phenytoin concentration in patients co-medicated with valproic acid. Ther Drug Monit 1989;11:134-9.

22. Kerrick JM, Wolff DL, Graves NM. Predicting unbound phenytoin concentrations in patients receiving valproic acid: a comparison of two prediction methods. Ann Pharmacother 1995;29:470-4.

23. Duncan JS, Patsalos PN, Shorvon SD. Effects of discontinuation of phenytoin, carbamazepine, and valproate on concomitant antiepileptic medication. Epilepsia 1991;32:101-15.

24. Salcman M, Defendini R, Correll J, Gilman S. Neuropathological changes in cerebellar biopsies of epileptic patients. Ann Neurol 1978;3:10-9.

25. Luef G, Chemelli A, Birbamer G, Aichner F, Bauer G. Phenytoin overdosage and cerebellar atrophy in epileptic patients: clinical and MRI findings. Eur Neurol 1994;34:79-81.

26. Koller WC, Glatt SL, Perlik S, Huckman MS, Fox JH. Cerebellar atrophy demonstrated by computed tomography. Neurology 1981;31:405-12.

27. Ney GC, Lantos G, Barr WB, Schaul N. Cerebellar atrophy in patients with long-term phenytoin exposure and epilepsy. Arch Neurol 1994;51:767-71.

28. De Marcos F, Ghizoni E, Kobayashi E, Li L, Cendes F. Cerebellar volume and long-term use of phenytoin. Seizure 2003;12:312-5.

29. Muñoz-Garcia D, Del Ser T, Bermejo F, Portera A. Truncal ataxia in chronic anticonvulsant treatment: association with drug-induced folate deficiency. J Neurol Sci 1982;55:305-11.

30. Hoffbrand A, Necheles T. Mechanism of folate deficiency in patients receiving phenytoin. Lancet 1968;292:528-30.

31. Maxwell J, Hunter J, Stewart D, Ardeman S, Williams R. Folate deficiency after anticonvulsant drugs: An effect of hepatic enzyme induction? Br Med J 1972;1:297-9. 